\title{
Cognitive Access Point to Handle Delay Sensitive Traffic in WLANs
}

\author{
Mazhar H Malik \\ Department of Computer Science \\ Universtia Ca Foscari \\ Venezia, Italy \\ Email:malik@dais.unive.it
}

\author{
Mehmet Aydin \\ Department of Computer Science \\ UWE, Frenchay \\ Bristol, UK \\ Email: mehmetaydin@acm.org
}

\author{
Qasim Awais \\ Department of Computer Science \\ University of Central Punjab \\ Lahore, Pakistan \\ Email: qasim.awais@ucp.edu.pk
}

\begin{abstract}
Over the last few years, the widespread use of wireless local area networks (WLANs) continues to gain more and more impetus. Due to the increase in variety of multimedia applications such as voice, video and gaming traffic, it is paramount to develop a mechanism for the quality of service $(\mathrm{QoS})$ to support different types of traffic in WLAN. IEEE 802.11e was introduced to handle delay sensitive traffic using Enhance Distributed Coordination Function (EDCF) which is an extension of Distributed Coordination Function (DCF) to categorize traffic in different groups e.g. real time and non real time traffic.

In this paper, We investigate the impact of traffic handling techniques as part of medium access control (MAC) on the performance of WLAN. Firstly, we propose MAC level parameters settings, which are found the most suitable to WLAN in comparison with DCF and EDCF and then we propose a cognitive access point (CAP) which is based on novel approaches to assign proprieties on station level using the proposed algorithm and embedded Packet fragmentation technique in the Revised MAC parameters in cognitive access point mechanism. The benefits of the proposed approach is validated via experimental results using OPNET Modeler 17.5.
\end{abstract}

Keywords-Wireless Local Networks, Cognitive Access Point, Packet fragmentation technique, EDCF.

\section{INTRODUCTION}

Cognitive radio networks [1] are communication networks of adaptive nodes, which can change their behaviors according to environment. In 1997, IEEE802.11x [2] was introduced as a set of standards set up based on Distributed Coordination Function (DCF) and Point Coordination Function (PCF). DCF is a mandatory component of the wireless networks, which supports nonreal time traffic while PCF is optional and only supports real time traffic in infrastructure based networks. Within this regard, IEEE802.11e [3], which is based on Enhance Distributed Coordination Function (EDCF), was introduced to overcome the QoS issue in wireless networks. Although there are various studies conducted to handle QoS problems including delay sensitive traffic problems, in this respect, it is expected that cognitive radio networks can help handling this sort of issues more efficiently. In this study, we propose a new scheme using a cognitive radio network approach and prove that this proposed scheme performs better than IEEE802.11e [4] to handle delay sensitive traffic.

There are a number of studies done on cognitive radio networks for handling delay sensitive traffic [1]. However, to the best knowledge of the authors, delay sensitive traffic in cognitive networks is considered in studies very rarely, and remained as not well-established research area, especially lacking of comprehensive experimental works.In [5] authors provide a heuristic control policy and large-delay-first channel allocation scheme along with improved policy using roll out algorithm, which partially considers cognitive radio networks that deal only with the proactive utilization of radio frequency. On the other hand, OPNET [6] is a network design and simulation tool to measure the performance of the networks, where it offers competitive advantages in the field of network design and mainly used in the Network Research and Development, design and simulation of the wireless networks.

In this paper, we analysis the performance of DCF and EDCF and then a revised EDCF is proposed with finetuned MAC parameters to achieve better performance. We introduce cognitive access points based on our novel approach in which priorities are assigned to Traffic Categories (TC) and station levels using the proposed algorithm. The proposed approach dynamically assign priorities to stations by measuring the traffic load of delay sensitive traffic.

The rest of the paper is organized as follows. In Section II we elaborate Coordination Functions in IEEE802.11 and QoS support in IEEE802.11e. In Section III the problems with IEEE802.11e are explained while Section IV explains the analytical model. In Section. $V$ provides the proposed novel approach in detail, Section VII presents implementation in simulation tool and analyze the results and. Section VIII overviews the study and indicates the final concluding remarks.

\section{EDCA AND QOS SUPPORT IN IEEE802.11E}

IEEE has introduced IEEE802.11e [7][8] standard in 2005 to facilitate handling the needs of QoS service in wireless communication networks. The standard introduced the concept of priority queue and transmission opportunities 
in backoff algorithm along with changes in the MAC layer. EDCA [9] suggests four different types of access categories, which are voice, video, best effort and background traffic. The purpose of the last category (background traffic) is to handle delay sensitive traffic proactively in comparison to DCF. EDCA imposes access priorities alongside access categories and handles the requests according to the priorities along with assignment of a contention window size which vary on the basis of type of traffic.

There are two types of queues; high level queue is the one applied before scheduling and low level queue, which happens after scheduling. Here, it is software based while on MAC hardware level, there are the following components: HCCA transmission, HCCA current Entry and HCCA Next entry.

The certain parameters [10] associated with EDCA are as follows;

1) Every Access category contains an Arbitrary inter frame spacing number (AIFSN), which is the time slot number after SIFS and can be explained using the AIFS=SIFS+AIFSN_Time Slot

2) AIFSN is always less than AIFS and it holds high priority and contention windows containing random numbers which are used in calculation of time interval and the backoff time, where Backoff time $=$ AIFS + Random $\left[\mathrm{CW}_{\min } ; \mathrm{CW}_{\max }\right]$

\section{A. Contention Windows: DCF vs EDCA}

As EDCA is designed to handle delay sensitive traffic, the contention window size for best effort and background remains the same while Voice and windows traffic change [5] as indicated in Table.1.

Table I: DCF and EDCA CW size difference

\begin{tabular}{|c|c|c|}
\hline Traffic Category & $\mathrm{CW}_{\min }$ & $\mathrm{CW}_{\max }$ \\
\hline $\mathrm{DCF}$ & $\mathrm{aCW}_{\min }$ & $\mathrm{aCW}_{\max }$ \\
\hline EDCA Voice & $\left(\mathrm{aCW}_{\min }+1\right) / 4-1$ & $\left(\mathrm{aCW}_{\min }+1\right) / 2-1$ \\
\hline EDCA Video & $\left(\mathrm{aCW}_{\min }+1\right) / 2-1$ & $\mathrm{aCW}_{\min }$ \\
\hline EDCA Best Effort & $\mathrm{aCW}_{\min }$ & $\mathrm{aCW}_{\max }$ \\
\hline EDCA Background & $\mathrm{aCW}_{\max }$ & $\mathrm{aCW}_{\max }$ \\
\hline
\end{tabular}

EDCF is designed to handle different types of QoS requirements with four different Access Categories (ACs), where each station contains of the channel access and starts backoff independently depending on its associated AC. Each access category use $\mathrm{CW}_{\min }[\mathrm{AC}], \mathrm{CW}_{\max }[\mathrm{AC}]$ and arbitration inter frame spacing (AIFS[AC]).

EDCF and DCF have the same contention method and every station has a frame to transmit, in need of waiting for the channel to be idle without interruption for a period AIFS [AC] and it start a random backoff process with its CW[AC]. When the channel becomes idle, the random backoff value is decremented and when it reaches to zero, the frame is transmitted. AIFS [AC] is calculated as in Eq. 1:

$$
A I F S[A C]=A I F S[A C] * \text { aSlotTime + aSIFSTime }
$$

AIFS value is defined according to the type of traffic, aSIFSTime is the duration of a short inter frame space, aSlotTime is time duration which backoff counter use as time unit that a station need to detect and the transmission of the frame from any other station and backoff time is calculated as follows:

$$
\text { backoff }- \text { time }=\text { random }_{\text {integer }} * \text { aSlotTime }
$$

Where random-integer is chosen in the range of $(0, C W[A C])$. Initial $\mathrm{CW}$ of each $\mathrm{AC}$ is $\mathrm{CW}_{\min }[\mathrm{AC}]$, when a collision happens, $\mathrm{CW}$ is doubled up to:

$$
C W_{\max }[A C]=2^{m} *\left(C W_{\min }[A C]\right)
$$

where $m$ is maximum backoff stage and when it reaches $\mathrm{CW}_{\max }$, it remain on that value until value is reset.

\section{PRoblems WITH IEEE802.11E}

In the process of contention control when every station in the standard has same priority level and station can only transmit data on the same priority level, which is far away from real world application as on station level the traffic can have different priorities so if the higher priority level traffic is at station level and station is handling traffic with same priority then it will lead to delay badly affecting the performance of the network.

In the fix priority assignment at station level TCP flow throughput will also be decreased because when in the phase of the TCP congestion avoidance, source waits to acknowledge and then sends the next packet so at station level if data is handled at the same priority level then it causes delay and leaves to decrease throughput.

\section{A. EDCA implementation Model}

When data reaches at MAC layer [11] of EDCA, it categories it according to the nature of data traffic and split it into four types of access categories which are shown in Figure. 1.

When data is in queue, the internal backoff calculates processing time and assigns priorities, which are defined for QoS, and arranges a queue for data processing, while Virtual Collision handler, which is basically CSMA/CA, ensures smoothness and accuracy in data and then the data is moved to the physical layer for further processing.

\section{ANALYTICAL MODEL}

For each access category, $A C_{i},(i=0, \ldots \ldots ., 3)$, let $W_{i, j}$ denote contention window size in $j^{\text {th }}$ backoff stage; i.e. after $j^{\text {th }}$ unsuccessful transmission; the contention windows will be minimum, $W_{i, 0}$. Let $J=m_{i}$ denote $j^{t h}$ backoff 


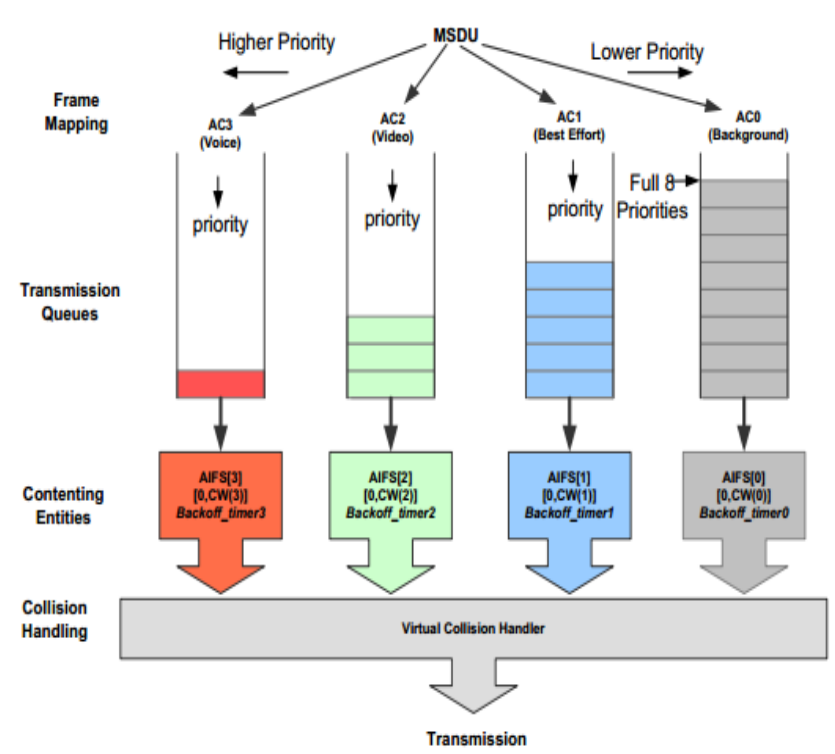

Figure 1: MAC layer of EDCA and Access Categories

stage where contention windows reaches at maximum size, $2^{m i} W_{i, 0}$. Also, let $L_{i}$ denote the retry limit of the retry counter;

$$
W_{i, j}= \begin{cases}2^{j} W_{i, 0} & j=0,1, \ldots, m_{i}-1 \\ 2^{m i} W_{i, 0} & j=m_{i}, \ldots, L_{i}\end{cases}
$$

Bi-dimensional Markov chain, $\left(S_{n}^{i}, B_{n}^{i}\right)$, is used to analyze EDCA behavior for $A C_{i}$, where the stochastic process of backoff stage is represented with $S_{n}^{i}$ and backoff counter with $B_{n}^{i}$. The state, $S_{n}^{i}=-1$, is the representation of the stage where backoff is empty and is considered as $S_{n}^{i}=0$. When backoff starts with a non-empty queue, the state space of Markov chain $\left(S_{n}^{i}, B_{n}^{i}\right)$ is defined as $(j, k)$, where $k=0,1, \ldots, W_{i, j}$ and $j=-1,0,1, \ldots, L_{i}$ and $W_{i,-1}=W_{i, 0}$. Figure. 2 shows the Markov chain for the priority class of $i$ of EDCF and transition probabilities are described in [12]. Let the steady state distribution of Markov chain be denoted by $b_{i, j, k}$ and suppose EDCA transmits when any of the states $(j, 0)$, where $j=0,1, \ldots, L_{i}$. As $b_{i, j, k}=p_{i}^{j} b_{i, 0,0}$ for $j=0,1, \ldots, L_{i}$, the probability of $\Pi_{i}$ will be:

$$
\Pi_{i}=\sum_{j=0}^{L_{i}}=b_{i, j, 0}=b_{i, 0,0} \frac{1-p_{i}^{L_{i}+1}}{1-p_{i}}
$$

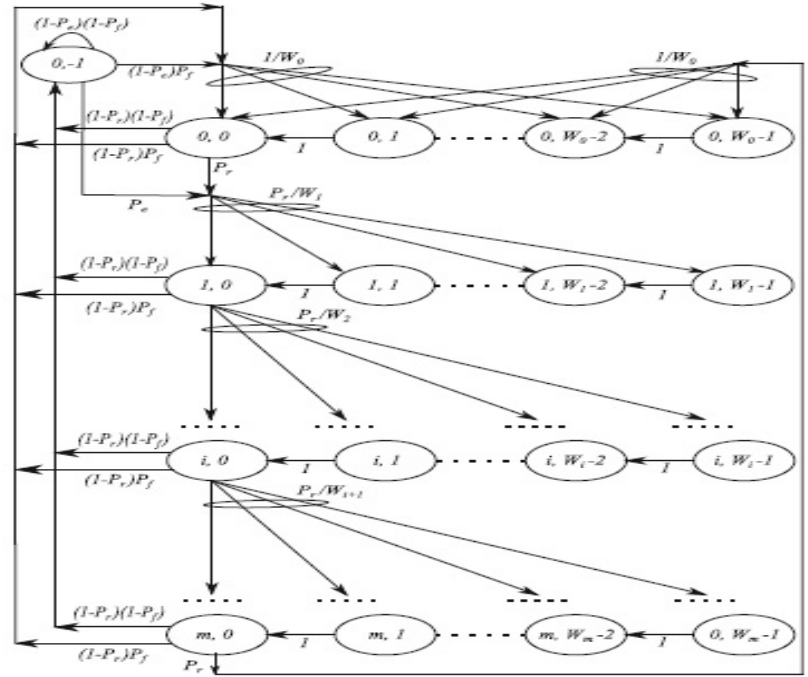

Figure 2: State transition diagram of the i class priority

where $\Pi_{i}$ can be expressed in Eq.6 as [12].

$$
\begin{array}{r}
\frac{1}{\tau_{i}}=\frac{\left(1-2 p_{i}^{*}\right)}{2\left(1-p_{i}^{*}\right)}+ \\
\frac{W_{i, 0}\left(\left(1-p_{i}\right)\left(1-\left(2 p_{i}\right)^{m i}\right)+\left(1-2 p_{i}\right)\left(2 p_{i}\right)^{m i}\left(1-p^{\left.L_{i}-m_{i}+1_{i}\right)}\right)\right.}{2\left(1-p_{i}^{*}\right)\left(1-2 p_{i}\right)\left(1-p^{\left.L_{i}+1_{i}\right)}\right.} \\
+\frac{1-p_{i}}{1-p^{L_{i}+1_{i}}} \frac{1-p_{i}}{q_{i}}\left(1+\frac{\left(W_{i, 0}-1\right) q_{i} p_{i}}{2\left(1-p_{i}^{*}\right)}\right) .
\end{array}
$$

The values of $p_{i}, p_{i}^{*}, q_{i}, p_{i}$ and $\rho_{i}^{*}$ can be estimated as following:

$p_{i}$ : when the station transmits data to more than one ACs, then virtual collision handler handles the queues and the probability of unsuccessful transmission is given as:

$$
p_{i}=1-\frac{1-p_{b}}{\prod_{c=0}^{i}(1-\tau)^{n i}} .
$$

while $p_{b}$ is the channel busy probability, which is calculated as:

$$
p_{b}=1-\prod_{i=0}^{N-1}(1-\tau)^{n i}
$$

$p_{i}^{*}$ is the probability that EDCA remains in the state of two transmission slots, which is:

$$
A_{i}=A I F S N[i]-A I S F N[N-1] .
$$

$q_{i}$ denotes the probability of the cases that, at least ,one packet arrives in state of $(-1,0)$. Here, the queue becomes non-empty and packet arrival rate is $\lambda_{i}$ for each EDCA $A C_{i}$, then $q_{i}$ is calculated as:

$$
q_{i}=\left(P_{s} e^{-\lambda, T_{s}}+\left(1-p_{b}\right) e^{-\lambda, T_{s}}+\left(p_{b}-p_{s}\right) e^{-\lambda, T_{c}}\right)
$$

where $T_{s}$ denotes a slot containing a successful transmission, $T_{e}$ is the real time duration of an empty slot and $T_{c}$ is 
a slot which contains two or more packets colliding as described in [12]. The probability of successful transmission is computed as:

$$
P_{s}=\prod_{i=0}^{N-1} n_{i} \tau_{i}\left(1-p_{i}\right)
$$

$q_{i}^{*}$ is the probability that packet arrives during countdown blocking and is expressed as:

$$
q_{i}^{*}=1-\frac{e^{-\lambda, T_{e}}\left(1-p_{i}^{*}\right.}{1-p_{i}^{*}\left(\frac{P_{s}}{P_{b}} e^{-\lambda, T_{s}}+\left(1-\frac{P_{s}}{P_{b}}\right) e^{-\lambda_{i}, T_{c}}\right)}
$$

$\rho_{i}$ : In [12] shown that $D_{i}^{S A T}$ is delay at MAC layer level and utilization factor $\rho_{i}$ is given by [13]:

$$
\rho_{i}=\min \left(1, \lambda_{i} D_{i}^{S A T}\right)
$$

$\rho_{i}^{*}$ : denotes the probability of such a case that there is a packet waiting in the queue of ECFA of AC $i$ when a transmission is completed, which is calculated with:

$$
1-\rho_{i}=P_{i}^{P B}\left(1-\rho_{i}^{*}\right)
$$

where $P_{i}^{P B}$ is the probability of not receiving any packet in the queue for transmission and performing a post-backoff procedure, while $P_{i}^{P B}$ can be expressed as:

$$
P_{i}^{P B}=\frac{1-\left(1-q_{i}^{*}\right)^{w_{i, 0}}}{w_{i, 0} q_{i}^{*}}
$$

\section{Proposed Novel Approach}

The cognitive access point has the functionality of priority assignment for not only on AC but also on station level. A station based priority assignment scheme is explained in Algorithm.1. A station based priority assignment scheme

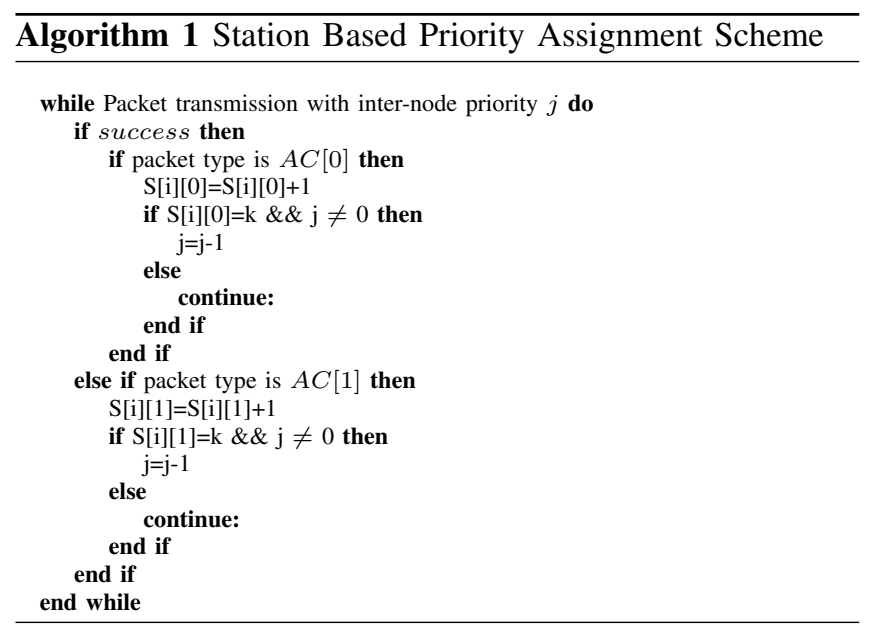

is explained in Algorithm.1. The detail of modification in EDCA method is explained below:

Decision factor $k$ expressing the limit to implement traffic

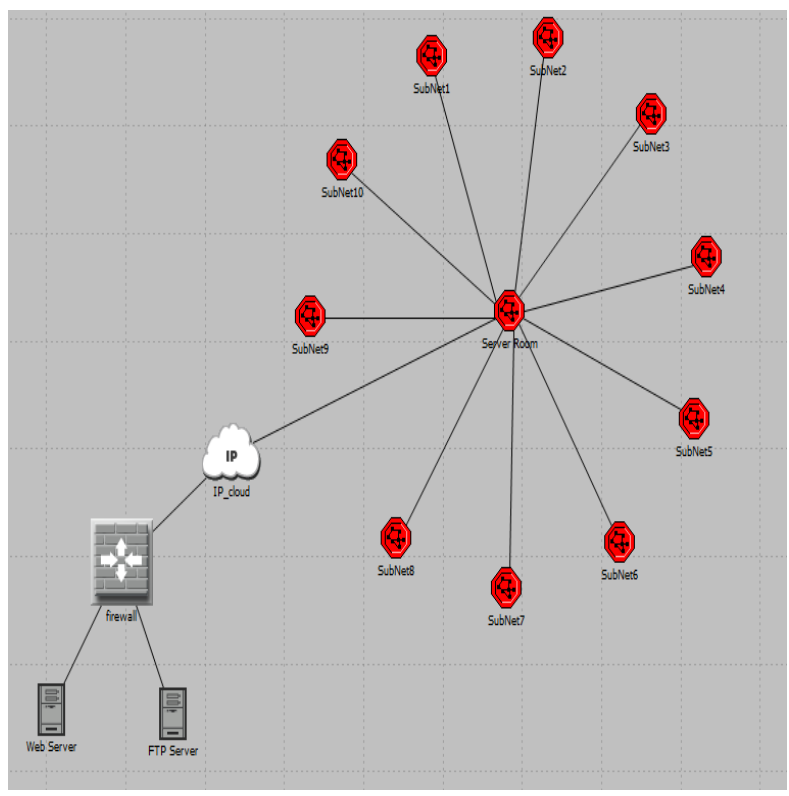

Figure 3: The Architecture of the Network designed

counter for each station along with traffic access category $\mathrm{AC}[0]$ and $\mathrm{AC}[1]$ and denotes by $\mathrm{S}[\mathrm{i}][0]$ and $\mathrm{S}[\mathrm{i}][1]$ for delay sensitive traffic. When both $\mathrm{S}[\mathrm{i}][0]$ and $\mathrm{S}[\mathrm{i}][1]$ traffic counters reach to the value of $k$, it will allow station more pertinent than other network stations. Then class $j$ will be increased to higher class $j-1$, which lead to assign higher priority and the flow control $\mathrm{S}[\mathrm{i}][\mathrm{x}]$ will be reset to zero by station.

\section{IMPLEMENTATION}

We consider a wireless network shown in Figure.3, which is deployed in a real world case, where E-learning system is implemented in a university and interconnecting different departments of the university. In this model,the network uses 10 access points and assumes that there are two types of user profiles which contain different types of applications and access categories, and the other which the type of applications can be run by particular profile according to their usage and access level; while centralize server room. It contains server and switch which are establishing communication with other access points, a firewall is deployed for which the network is facilitated to connect external cloud to get access to FTP and web server, where the web server holds database and can be accessed locally and from home.The implementation scenario has the following properties;

1) In the first scenario, we implemented Access Points using ordinary EDCA configuration and associated with ordinary stations

2) In the second scenario, we implemented Access Points with revised configuration called Cognitive Access point along with Priority Traffic Concept on station level called CognetSTA. 
Table. 5 shows revised parameters which are used for Video and audio traffic in multimedia traffic model.

Table II: Cognitive Access Point and CogSTA Revised Parameters

\begin{tabular}{|c|c|c|c|}
\hline Traffic Type & CWmin & CWmax & AIFSN \\
\hline Audio & 7 & 15 & 2 \\
\hline Video & 15 & 31 & 2 \\
\hline Best Effort & 31 & 63 & 3 \\
\hline Background & 63 & 1023 & 7 \\
\hline
\end{tabular}

\section{Comparative AnALysis}

In this section, we analyze the impact of our proposed MAC configuration parameters with default access points and with our proposed Cognitive Access points. We measure the performance of the network using QoS parameters, e.g. Network delay, MAC delay, Throughput, Queue size and packet retransmission ratio. Figure. 4 shows the throughput using default parameters is in the range of $300 \mathrm{bits} / \mathrm{sec}$ and remain constant in the same range while using revised parameters the throughput is increased and it is more or less $350 \mathrm{bits} / \mathrm{sec}$, which achieves about $15 \%$ better performance in comparison to original EDCA parameters. While the proposed cognitive access point technique results show that throughput is higher than $400 \mathrm{bits} / \mathrm{sec}$ and in increasing trend.

We analyze two types of delays in the network; (i) we analyze the MAC delay in which the time taken to enter data in MAC layer and to pass it out from the MAC layer, (ii) we measure the total delay of the system from source to destination, which is also called End-to-End delay. Figure.5 and Figure 6 display MAC and network delays, accordingly. Apparently, the proposed scheme causes much less MAC delay approximating to zero, which seems very less in comparison to default EDCA, while altering MAC parameters also lead to good results as compared with default EDCA. The level of delay achieved with altered MAC parameters is between 0.001 to 0.0015 while the option with default parameters also succeeds with very good performance of around 0.002 . Overall network delay remains within the range of 0.35 to 0.4 using default parameters while it is significantly dropped to 0.05 using the proposed parameters and it is further dropped to the range of 0.0005 to 0.0007 using our proposed cognitive access point technique, which seems approximated to zero . Figure.7 shows the queue length at access points, where the queue length of EDCA with default parameters are 12 to 14 packets per second, while it is dropped to 8 to 10 packets per second with proposed new parameters,. The use of cognitive access point technique helps achieve further decrease in queue lengths dropping it to 6 packets per second. One of the network performance measures used is the level of packet

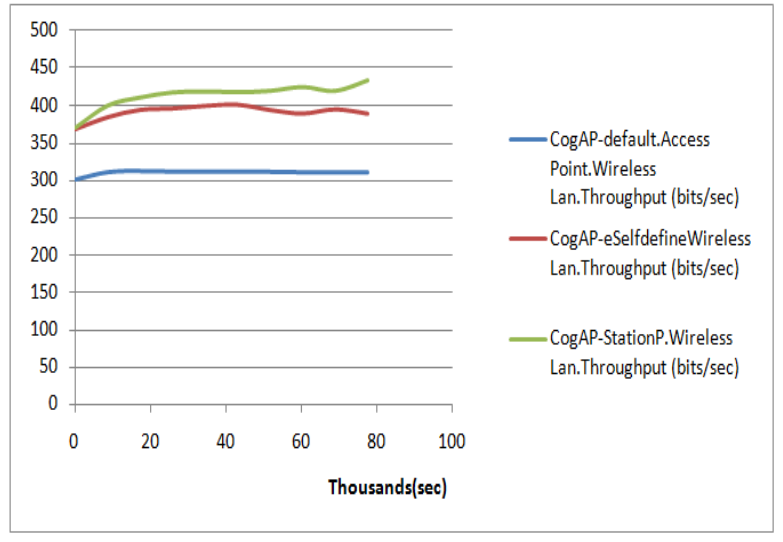

Figure 4: EDCA-Default,EDCA-Selfdefine and Cognitive Access point Throughput

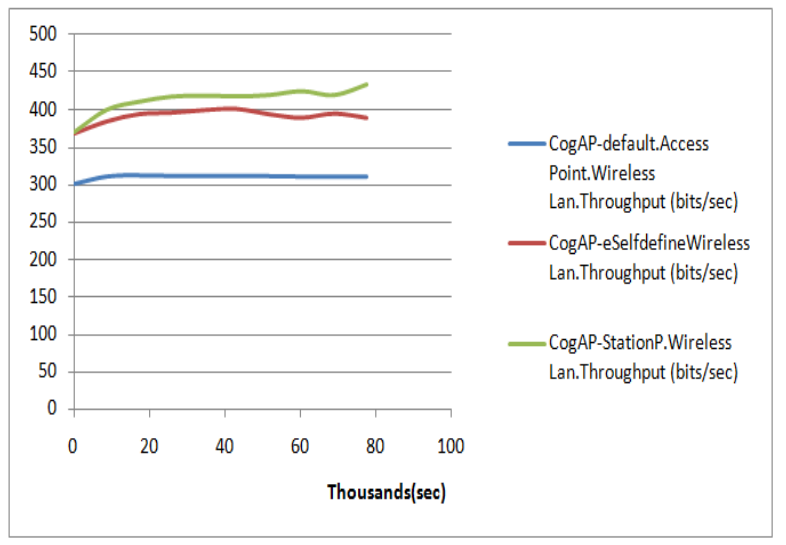

Figure 5: EDCA-Default,EDCA-Selfdefine and Cognitive Access point MAC Delay

loss. Obviously, a re-transmission operations is required whenever any packet loss happens. Figure 8 indicates that the altered MAC parameter set provides 50 percent less packet retransmission, which means that this scheme results in very less packet loss while the proposed cognitive access point supports the network further for not losing packets on the way, therefore the need for retransmission is reduced near to 0.001 . That proves that the proposed approach offers very good QoS.

\section{CONCLUSION}

In this paper, firstly we proposed a revised set of parameters for IEEE802.11e standard, which are suitable for all types of network implementation and our results are validated using simulation. Secondly, we propose a Cognitive Access point which have the functionality of a computing traffic load according to Access categories and assigning priority to stations which have high flow of particular AC and is determined by a $k$ factor. This factor is a threshold value used when $\mathrm{AC}$ of the particular station reaches to 


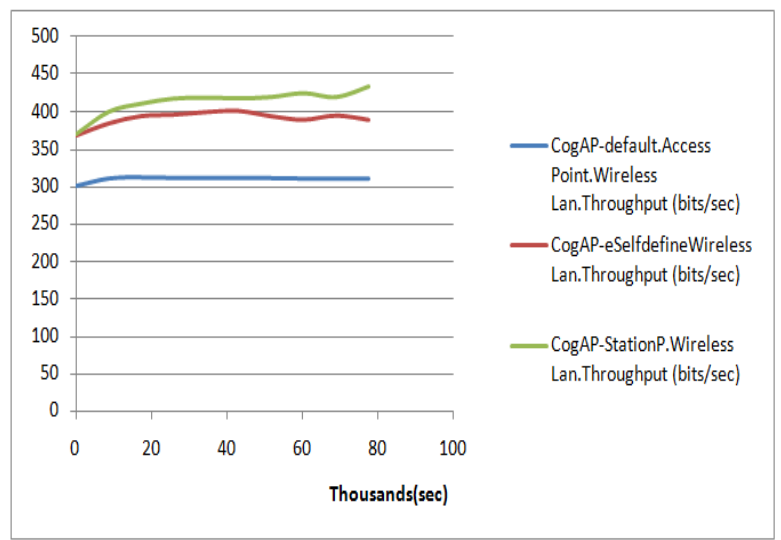

Figure 6: EDCA-Default,EDCA-Selfdefine and Cognitive Access point Network Delay

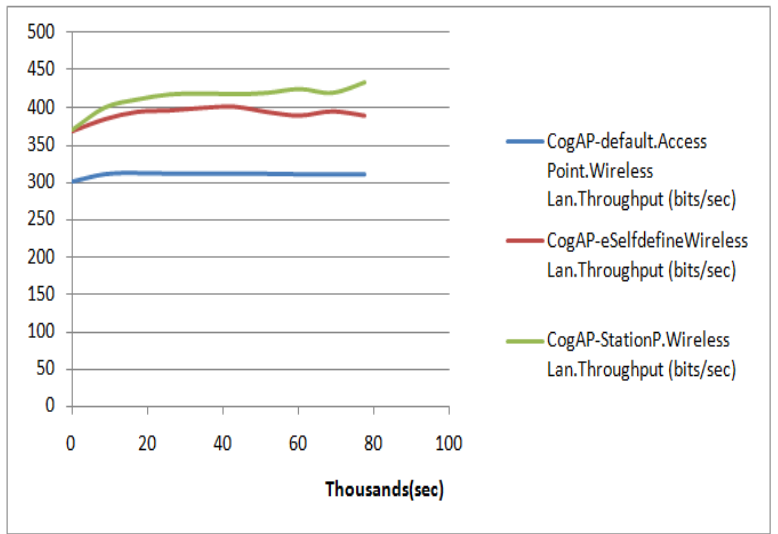

Figure 7: EDCA-Default,EDCA-Selfdefine and Cognitive Access point Queue Size

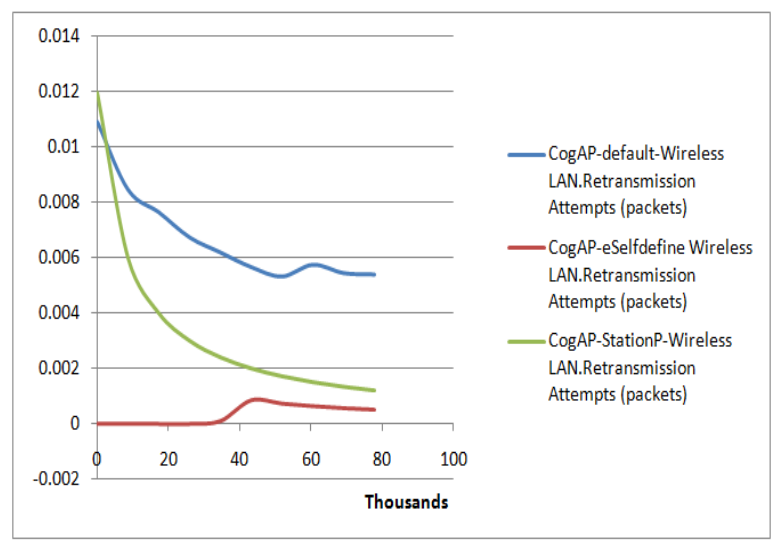

Figure 8: EDCA-Default,EDCA-Selfdefine and Cognitive Access Point Retransmission that $k$ value, the proposed cognitive access point decreases AIFSN for that particular access category and the priority of corresponding traffic category will be increased for that particular station. The performance of our proposed approach including altered parameters and cognitive access points is validated using simulation and statistics show that our approach is performing proactive with respect to Throughput, Network and MAC delay and Queue size. Further aspects of the network performance such as energy management will be considered for further studies in the future using cognitive access points to handle traffic in routing level as well.

\section{REFERENCES}

[1] C. Fortuna and M. Mohorcic, "Trends in the development of communication networks: Cognitive networks," Computer Networks, vol. 53, no. 9, pp. 1354-1376, 2009.

[2] W. Stallings, Wireless Communications \& Networks, 2/E. Pearson Education India, 2009.

[3] S. Choi, J. Del Prado, S. Mangold et al., "Ieee 802.11 e contention-based channel access (edcf) performance evaluation,' in Communications, 2003. ICC'03. IEEE International Conference on, vol. 2. IEEE, 2003, pp. 1151-1156.

[4] A. Grilo, M. Macedo, and M. Nunes, "A scheduling algorithm for qos support in ieee802. 11 networks," Wireless Communications, IEEE, vol. 10, no. 3, pp. 36-43, 2003.

[5] F. Peng, H. M. Alnuweiri, and V. C. Leung, "Analysis of burst transmission in ieee 802.11 e wireless lans," in Communications, 2006. ICC'06. IEEE International Conference on, vol. 2. IEEE, 2006, pp. 535-539.

[6] J. Prokkola, "Opnet-network simulator," URL http://www. telecomlab. oulu. fi/kurssit/521365A tietoliikennetekniikan simuloinnit ja tyokalut/Opnet esittely, vol. 7, 2006.

[7] Y. Xiao, "Enhanced dcf of ieee 802.11 e to support qos," in Wireless Communications and Networking, 2003. WCNC 2003. 2003 IEEE, vol. 2. IEEE, 2003, pp. 1291-1296.

[8] X. Yang, "Ieee 802.11 e: Qos provisioning at the mac layer," Wireless Communications, IEEE, vol. 11, no. 3, pp. 72-79, 2004.

[9] T. Larsson and Y. Liu, "A study of edca and def in multihop ad hoc networks," Sweden, March, vol. 13, 2008.

[10] H. Zhai, X. Chen, and Y. Fang, "How well can the ieee 802.11 wireless lan support quality of service?" Wireless Communications, IEEE Transactions on, vol. 4, no. 6, pp. 3084-3094, 2005.

[11] S. Mangold, S. Choi, P. May, O. Klein, G. Hiertz, and L. Stibor, "Ieee 802.11 e wireless lan for quality of service," in Proc. European Wireless, vol. 2, 2002, pp. 32-39.

[12] P. E. Engelstad and O. Osterbo, "Analysis of the total delay of ieee 802.11 e edca and 802.11 dcf," in Communications, 2006. ICC'06. IEEE International Conference on, vol. 2. IEEE, 2006, pp. 552-559.

[13] L. Kleinrock, Theory, volume 1, Queueing systems. Wileyinterscience, 1975. 